\title{
Evaluating Protection for External Factors in Multi-State Systems
}

\author{
Hongyan Dui*
}

School of Management Engineering, Zhengzhou University, Zhengzhou, 450001, China

\begin{abstract}
An external factor is an added condition beyond the normal load of the system affecting the system's success. Each external factor has an influence on system components, which may lead to system failure. To decrease the effects of different states of the external factors on system components, multistate protections are established to protect the components from the effects of the external factors. The protection states are dependent on the external factor state. When a protection is at different states, the protection may be damaged. In this paper, considering the joint effect of the external factors and protections on system components, a measure of states of the protections on the system reliability is introduced to identify which protection level has the most important influence on the system success. Based on the importance values of different protections, appropriate actions can be applied in system components to improve the system protection and reliability and reduce the effects of external factors. At last, an example of a computer server system is used to demonstrate the proposed method.
\end{abstract}

Keywords: system components; multistate protections; external factions; system reliability

(Submitted on November 19, 2017; First revised on November 23, 2017; Second revised on December 8, 2017; Accepted on December 14, 2017)

(C) 2018 Totem Publisher, Inc. All rights reserved.

\section{Notations}

$x_{i}(t) \quad$ state of component $i$ at time $t, x_{i}(t)=0,1,2, \ldots, M_{i}$

$e_{i} \quad$ index of external factor $e_{i}$

$x_{e_{i}}(t) \quad$ state of external factor $e_{i}$ at time $t, x_{e_{i}}(t)=0,1, \ldots, s_{i}$

$p l_{i} \quad$ index of protective layer $p l_{i}$

$x_{p l_{i}}(t) \quad$ state of protective layer $p l_{i}$ at time $t, x_{p l_{i}}(t)=0,1, \ldots, s_{i}$

$v_{p l_{i} q} \quad$ damaged probability of state $q$ of protective layer $p l_{i}$

$d \quad$ state threshold value of a component or system

$S_{k}(i, t) \quad$ importance of state $q$ of protective layer $p l_{i}$

$X(t) \quad\left(x_{1}(t), x_{2}(t), \ldots, x_{n}(t)\right):$ state vector of the components

$\Phi(X(t)) \quad$ system structure function, $\Phi(X(t))=\Phi\left(x_{1}(t), x_{2}(t), \ldots, x_{n}(t)\right)$ 


\section{Introduction}

An external factor is an added condition beyond the normal load of the system affecting the success of the system. The external factors have effects on the system reliability $[2,6]$. In multi-state systems, the external factors have multiple states, which have a different influence on the components. For example, Si et al. [7] analyzed the effect of the external factor state on the system performance. Hausken and Levitin [4] reviewed the system defense and attack models. Zhai et al. [10] analyzed the defense and attack based on the sharing performance. Xing and Levitin [9] gave a data protection policy based on the partition technique where sensitive data are divided and distributed. Di et al. [1] analyzed the object defense with preventive strike and false targets.

To decrease the effect of external factors on components and system, a protection model should be built to protect the components and system. Considering the separation and protection of system elements, Levitin and Ben-Haim [5] presented a generalized model of damage by intentional attack. Taranto et al. [8] analyzed the importance on voltage protection in active distribution networks of voltage security. Dui et al. [3] proposed a new importance measure to evaluate the degree of the impact of external factors on the system.

According to the external factor states, the protection has multiple states. In such protections, different protection states have effects on the system's reliability. The states of the protections are dependent on the states of the external factors. It is important to identify which protection state has the greatest effect on the system reliability.

In this paper, the protections for external factors are evaluated. Then, an importance measure of protection states is introduced to identify which protection state has the most effect on the system's reliability. Considering the conditional probabilities, a Bayesian network (BN) based method is used to calculate the importance. At last, a numerical example is used to illustrate the use of the proposed methods.

\section{Protection for external factors}

In multistate systems, each external factor has an influence on components or system. Thus, we should build the protective layers to protect the components, in which protective layer $i$ can protect component $i$. For example, a multistate system with two external factors is shown in Figure 1. Components 1 and 2 are impacted by external factors 1 and 2 . To protect the components and system from the effects by external factors, we use protective layers 1 , 2, and 3 to protect each component and system. In Figure 1, the directed edges represent the conditional dependency between an external factor and a component, and component and the system.

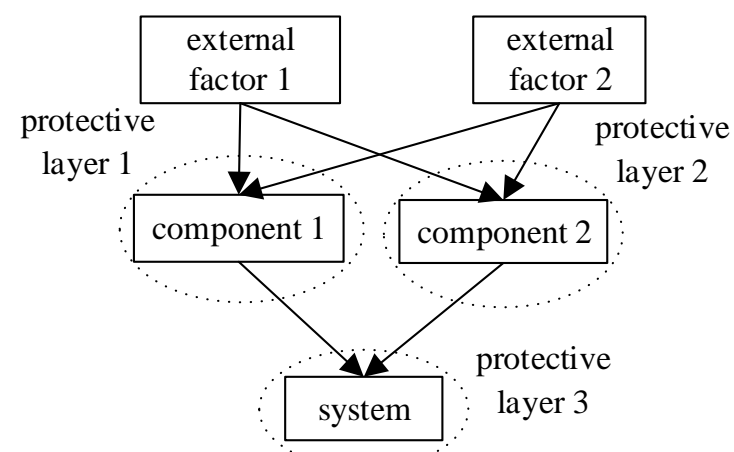

Figure 1. An example of two components with external factors

External factor $e_{i}$ has $s_{i}+1$ states, in which 0 is the complete failure states, and $s_{i}$ is the perfect working state. Protective layer $p l_{i}$ is used to prevent the external factor $e_{i}$ from impacting component $i$. Each state of external factors can impact components. When external factor $e_{i}$ is at state $s_{i}$, the external factor has the biggest influence on component $i$. When external factor $e_{i}$ is at state 0 , the external factor has no influence on component $i$. Thus, the states of protective layer $p l_{i}$ can protect the states of external factor $e_{i}$, and the states of protective layer $p l_{i}$ are the same as the states of external factor $e_{i}$. When protective layer $p l_{i}$ is at state 0 , the protective layer $p l_{i}$ completely fails and does not work. In this situation, 
external factor $e_{i}$ perfectly works. Thus, when state $s_{i}$ of external factor $e_{i}$ has an influence on component $i$, the protective layer $p l_{i}$ is at state 0 . Similarly, when state 0 of external factor $e_{i}$ has an influence on component $i$, the protective layer $p l_{i}$ is at state $s_{i}$. Then when state $l$ of external factor $e_{i}$ has an influence on component $i$, the protective layer $p l_{i}$ is at state $s_{i}-l$

When the protective layers are attacked, they can be damaged. We use $v_{p l_{i q}}$ to represent the damaged probability of state $q$ of protective layer $p l_{i}$. When the protective layers have been damaged, the external factors will affect the components. On the contrary, when the protective layers are working, the external factors have no effect on the components. Then, the probability that component $i$ is at state $m$ when protective layer $p l_{i}$ is at state $q$ is in equation (1).

$$
\operatorname{Pr}\left\{x_{i}(t)=m \mid x_{p l_{i}}(t)=q\right\}=\operatorname{Pr}\left\{x_{i}(t)=m \mid x_{e_{i}}(t)=s_{i}-q\right\} \cdot v_{p l_{i} q}+\operatorname{Pr}\left\{x_{i}(t)=m \mid x_{e_{i}}(t)=0\right\} \cdot\left(1-v_{p l_{i} q}\right)
$$

Then when component $i$ is at state $m$, we have

$$
\begin{aligned}
& \operatorname{Pr}\left\{x_{i}(t)=m\right\}=\sum_{q=0}^{s_{i}} \operatorname{Pr}\left\{x_{i}(t)=m \mid x_{p l_{i}}(t)=q\right\} \cdot \operatorname{Pr}\left\{x_{p l_{i}}(t)=q\right\} \\
& =\sum_{q=0}^{s_{i}} \operatorname{Pr}\left\{x_{p l_{i}}(t)=q\right\}\left[\operatorname{Pr}\left\{x_{i}(t)=m \mid x_{e_{i}}(t)=s_{i}-q\right\} \cdot v_{p l_{i} q}+\operatorname{Pr}\left\{x_{i}(t)=m \mid x_{e_{i}}(t)=0\right\} \cdot\left(1-v_{p l_{i}}\right)\right] .
\end{aligned}
$$

Thus, the effect of state $q$ of protective layer $p l_{i}$ on the state $m$ of component $i$ is

$$
\frac{\partial \operatorname{Pr}\left\{x_{i}(t)=m\right\}}{\partial \operatorname{Pr}\left\{x_{p l_{i}}(t)=q\right\}}=\operatorname{Pr}\left\{x_{i}(t)=m \mid x_{e_{i}}(t)=s_{i}-q\right\} \cdot v_{p l_{i} q}+\operatorname{Pr}\left\{x_{i}(t)=m \mid x_{e_{i}}(t)=0\right\} \cdot\left(1-v_{p l l_{i} q}\right) .
$$

We use $d$ to represent the state threshold value of a component or system, i.e. when the system state is $\Phi(X(t)) \geq d$ or component state $x_{i}(t) \geq d$, the system or component $i$ works. Then, the importance of state $q$ of protective layer $p l_{i}$ is

$$
\begin{aligned}
& S_{k}(i, t)=\frac{\partial \operatorname{Pr}\{\Phi(X(t)) \geq d\}}{\partial \operatorname{Pr}\left\{x_{p l_{i}}(t)=q\right\}}=\frac{\partial \operatorname{Pr}\{\Phi(X(t)) \geq d\}}{\partial \operatorname{Pr}\left\{x_{i}(t) \geq d\right\}} \cdot \frac{\partial \operatorname{Pr}\left\{x_{i}(t) \geq d\right\}}{\partial \operatorname{Pr}\left\{x_{p l_{i}}(t)=q\right\}} \\
& =\frac{\partial \operatorname{Pr}\{\Phi(X(t)) \geq d\}}{\partial \operatorname{Pr}\left\{x_{i}(t) \geq d\right\}} \cdot\left[\operatorname{Pr}\left\{x_{i}(t) \geq d \mid x_{e_{i}}(t)=s_{i}-q\right\} \cdot v_{p l_{i} q}+\operatorname{Pr}\left\{x_{i}(t) \geq d \mid x_{e_{i}}(t)=0\right\} \cdot\left(1-v_{p l_{i} q}\right)\right] .
\end{aligned}
$$

The importance $S_{k}(i, t)$ is the effect of state $q$ of protective layer $p l_{i}$ on the system's reliability. Equation (4) can be used to identify which protection state has the most effect on the system's reliability.

In equation (4), there are some conditional probabilities $\operatorname{Pr}\left\{x_{i}(t) \geq d \mid x_{e_{i}}(t)=s_{i}-q\right\}$ and $\operatorname{Pr}\left\{x_{i}(t) \geq d \mid x_{e_{i}}(t)=0\right\}$ that we can use the $\mathrm{BN}$ to calculate the importance $S_{k}(i, t)$. In BN considering the effect of external factors, nodes represent the components, system and external factors. Edges represent conditional dependencies between external factors and components, pointing to the component nodes from the external factor nodes. These nodes are not connected directly by edges, but are conditionally independent of each other. Each node has a conditional probability table to express the interdependence between nodes quantitatively. For example, $\operatorname{Pr}\left\{x_{i}(t) \geq d \mid x_{e_{i}}(t)=s_{i}-q\right\}$ represents the conditional probability distribution of component state to describe the relationship intensity with its father variable (external factor states). Child nodes take the values of parent nodes as input to generate their conditional probability tables. Some nodes that have no parent nodes are called root nodes whose conditional probability tables are determined by prior probability. For example, if an external factor 
node becomes a root variable, its state probability distribution is called as prior probability distribution.

When considering the effect of external factors, the component variables and external factor variables describe all the fractional parts of a certain system. The detailed descriptions are as follows.

(1) The system state variables can use the structure function to describe the probability distributions. Components are statistically independent, so

$$
\begin{aligned}
& \operatorname{Pr}\left(\Phi\left(m_{i}, X(t)\right) \geq d\right)=\sum_{X} \operatorname{Pr}\left(\Phi\left(m_{i}, X(t)\right) \geq d \mid x_{1}(t), \ldots x_{i-1}(t), m, x_{i+1}(t), \ldots, x_{n}(t)\right) \cdot \operatorname{Pr}\left(x_{1}(t), \ldots x_{i-1}(t), m, x_{i+1}(t), \ldots, x_{n}(t)\right) \\
& =\sum_{X(t)} \operatorname{Pr}\left(\Phi\left(m_{i}, X(t)\right) \geq d \mid x_{1}(t), \ldots x_{i-1}(t), m, x_{i+1}(t), \ldots, x_{n}(t)\right) \operatorname{Pr}\left(x_{1}(t)\right) \ldots \operatorname{Pr}\left(x_{i-1}(t)\right) \operatorname{Pr}\left(x_{i}(t)=m\right) \operatorname{Pr}\left(x_{i+1}(t)\right) \ldots \operatorname{Pr}\left(x_{n}(t)\right) .
\end{aligned}
$$

$\operatorname{Pr}\left(\Phi\left(m_{i}, X(t)\right) \geq d \mid x_{1}(t), \ldots x_{i-1}(t), m, x_{i+1}(t), \ldots, x_{n}(t)\right)$ can be got by the system structure. Then we have $\operatorname{Pr}\{\Phi(X(t)) \geq d\}=\sum_{m=0}^{M} \operatorname{Pr}\left(x_{i}(t)=m\right) \cdot \operatorname{Pr}\left(\Phi\left(m_{i}, X(t)\right) \geq d\right)$.

(2) External factors are statistically independent, so we have

$$
\begin{aligned}
& \operatorname{Pr}\left(x_{i}(t) \geq d \mid x_{e_{i}}(t)=j\right)=\sum_{X_{e}} \operatorname{Pr}\left(x_{e_{1}}(t), \ldots, x_{e_{i-1}}(t), j, x_{e_{i+1}}(t), \ldots, x_{e_{N}}(t)\right) \operatorname{Pr}\left(x_{i}(t) \geq d \mid\left(x_{e_{1}}(t), \ldots, x_{e_{i-1}}(t), j, x_{e_{i+1}}(t), \ldots, x_{e_{N}}(t)\right)\right) \\
& =\sum_{x_{e}} \operatorname{Pr}\left(x_{e_{1}}(t)\right) \ldots \operatorname{Pr}\left(x_{e_{i-1}}(t)\right) \operatorname{Pr}\left(x_{e_{i}}(t)=j\right) \operatorname{Pr}\left(x_{e_{i+1}}(t)\right) \ldots \operatorname{Pr}\left(x_{e_{N}}(t)\right) \operatorname{Pr}\left(x_{i}(t) \geq d \mid\left(x_{e_{1}}(t), \ldots, x_{e_{e_{i-1}}}(t), j, x_{e_{e_{i+1}}}(t), \ldots, x_{e_{N}}(t)\right)\right) .
\end{aligned}
$$

$\operatorname{Pr}\left(x_{e_{i}}(t)\right)$ can be got by the direct information of external factors. $\operatorname{Pr}\left(x_{i}(t) \geq d \mid\left(x_{e_{1}}(t), \ldots, x_{e_{i-1}}(t), j, x_{e_{i+1}}(t), \ldots, x_{e_{N}}(t)\right)\right)$ can be determined by the inference information of external factors. So, $\operatorname{Pr}\left(x_{i}(t) \geq d \mid x_{e_{i}}(t)=j\right)$ can be calculated. Then according to equation (4), the proposed importance can be got.

\section{Numerical example}

In this section, we use an example of a computer server system to demonstrate the proposed method. In Figure 2, a system consists of three computer servers. Each computer server can be protected by the corresponding radar. The computer servers can be assaulted by one or two anti-aircrafts. When no anti-aircrafts assault, the state is in working state 0 . When one antiaircraft assaults, the state is in intermediate state 1 . When two anti-aircrafts assault, the state is in complete failure state 2 . The damaged probabilities of radars are $\operatorname{Pr}\left\{x_{p l_{i}}(t)=0\right\}=0.2, \operatorname{Pr}\left\{x_{p l_{i}}(t)=1\right\}=0.7, \operatorname{Pr}\left\{x_{p l_{i}}(t)=2\right\}=0.1$.

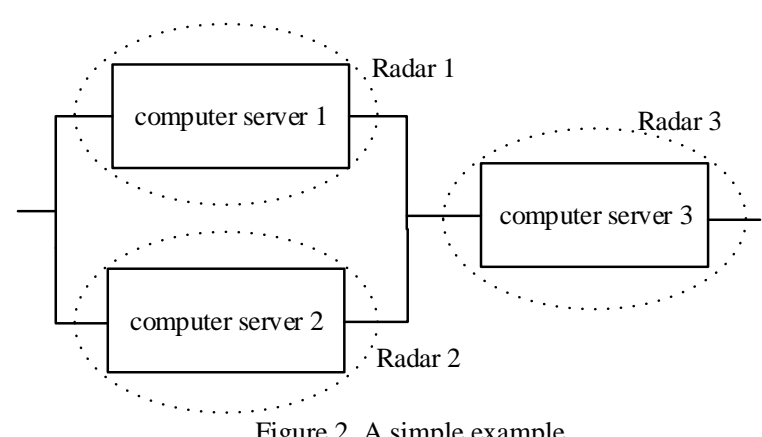

Figure 2. A simple example

The computer servers follows the exponential distributions. When the state is in working state 0 , we assume $\lambda_{1}=2.5$, $\lambda_{2}=7.1, \lambda_{3}=11.5$, and $\operatorname{Pr}\left\{x_{i}(t)=1 \mid x_{e_{1}}(t)=0\right\}=e^{-\lambda_{i} t}$. When the state is in intermediate state 1 , we assume $\lambda_{1}=5$, $\lambda_{2}=8.5, \lambda_{3}=1.3$, and $\operatorname{Pr}\left\{x_{i}(t)=1 \mid x_{e_{1}}(t)=1\right\}=e^{-\lambda_{i} t}$. When the state is in complete failure state, we have $\operatorname{Pr}\left\{x_{i}(t)=1 \mid x_{e_{1}}(t)=2\right\}=0$. The damaged probabilities of radars are $v_{p l_{1} 0}=1, v_{p l_{1}}=0.6, v_{p l_{1} 2}=0.3, v_{p l_{1} 0}=1, v_{p l_{2} 1}=0.4, v_{p l_{2} 2}=0.2$, 
$v_{p l_{3} 0}=1, v_{p l_{3} 1}=0.2, v_{p l_{3} 2}=0.05$. The importance $S_{k}(i, t)$ of protective state of computer server $i$ is as in Figure 3 .

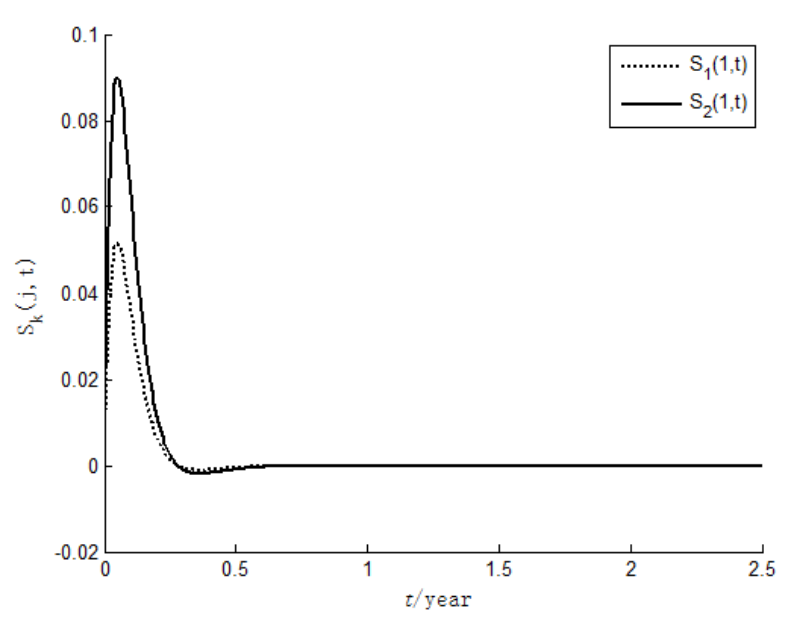

(a) The importance $S_{k}(1, t)$

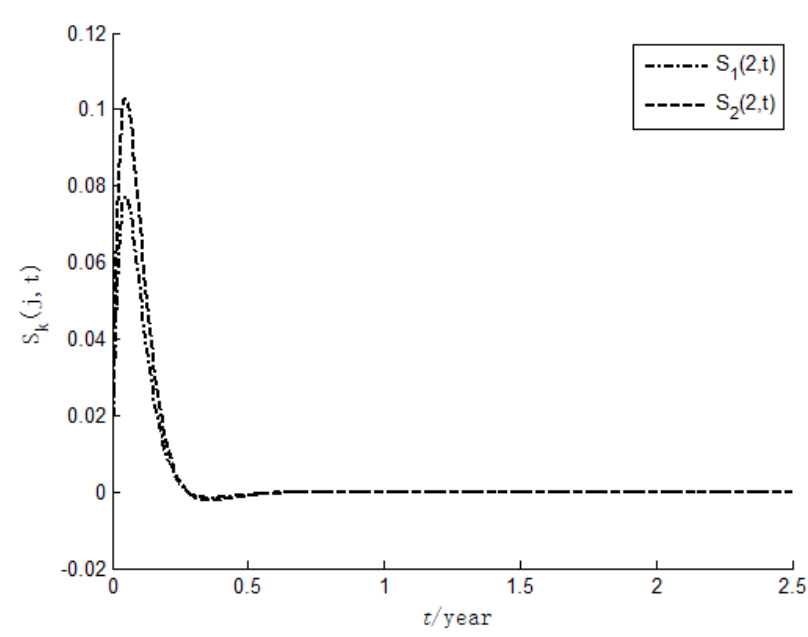

(b) The importance $S_{k}(2, t)$

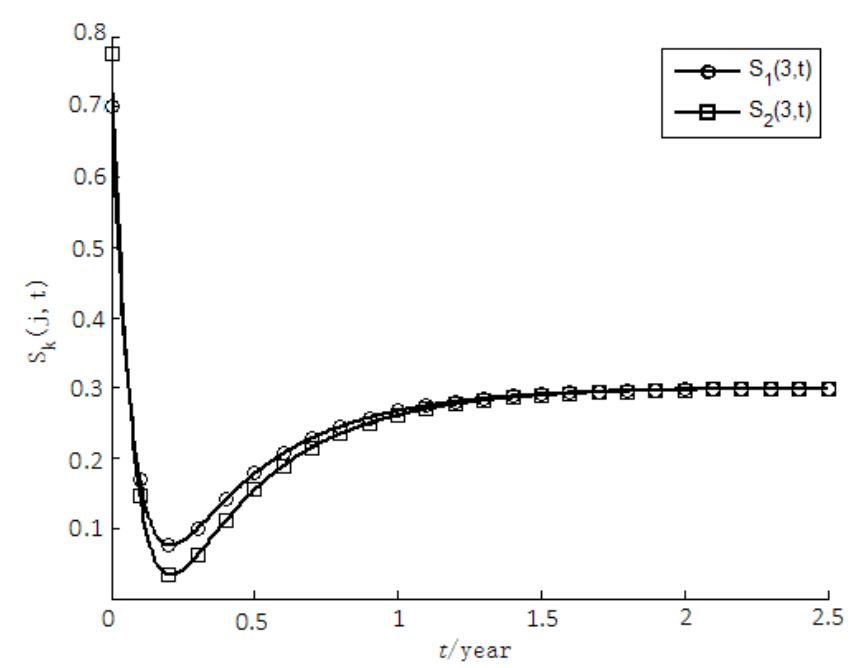

(c) The importance $S_{k}(3, t)$

Figure 3. The importance values of protective state of computer server $i$

According to equation (4), we can get

$$
S_{k}(1, t)=\operatorname{Pr}\left\{x_{3}(t)=1\right\} \operatorname{Pr}\left\{x_{2}(t)=0\right\}\left[v_{p l_{1} k} \operatorname{Pr}\left\{x_{1}(t)=1 \mid x_{e c_{1}}(t)=2-k\right\}+\left(1-v_{p l_{1} k}\right) \operatorname{Pr}\left\{x_{1}(t)=1 \mid x_{e c_{1}}(t)=0\right\}\right],
$$

and $\operatorname{Pr}\left\{x_{3}(t)=1\right\}=0.14 e^{-13 t}+0.66 e^{-11.5 t}$. Thus, when $t>0.3, \operatorname{Pr}\left\{x_{3}(t)=1\right\}$ is almost 0 . Thus, the effects of protection state of computer server 1 is close to 0 with time, which is similar for computer server 2 . $S_{2}(1, t)=\operatorname{Pr}\left\{x_{3}(t)=1\right\} \operatorname{Pr}\left\{x_{2}(t)=0\right\} e^{-2.5 t}$, and $S_{1}(1, t)=\operatorname{Pr}\left\{x_{3}(t)=1\right\} \operatorname{Pr}\left\{x_{2}(t)=0\right\} 0.6 e^{-5 t}+0.4 e^{-2.5 t}$. Then we have $S_{2}(1, t)>S_{1}(1, t)$, as shown in Figure 3(a). Similarly, we can get $S_{2}(2, t)>S_{1}(2, t)$, as shown in Figure 3(b). $S_{2}(3, t)=1-\operatorname{Pr}\left\{x_{1}(t)=0\right\} \operatorname{Pr}\left\{x_{2}(t)=0\right\} e^{-11.5 t}$, and $S_{1}(3, t)=1-\operatorname{Pr}\left\{x_{1}(t)=0\right\} \operatorname{Pr}\left\{x_{2}(t)=0\right\} \quad 0.2 e^{-1.3 t}+0.8 e^{-11.5 t}$.

Then we have $S_{2}(3, t)<S_{1}(3, t)$, as shown in Figure 3(c).

\section{Conclusions}

This paper has analyzed the protection for the effects of the external factor. The probability of component state changes with the appearance of external factor, and the protection for external factor can decrease its effect on the component. Thus, the system reliability will be also influenced. The proposed importance can identify the most important protection state for 
improving the system's reliability. In different life phase, the importance ranks of the protection levels are different.

\section{Acknowledgements}

This work was supported by the National Natural Science Foundation of China (Nos. 71501173, 71571168).

\section{References}

1. W. Di, X. Hui, and P. Rui, "Object defense with preventive strike and false targets", Reliability Engineering \& System Safety, vol. 169, pp. 76-80, 2018

2. H. Dui, L. Chen, and S. Wu, "Generalized integrated importance measure for system performance evaluation: application to a propeller plane system”, Eksploatacja i Niezawodnosc-Maintenance and Reliability, vol. 19, pp. 279-286, 2017.

3. H. Dui, S. Si, S. Wu, and C. M. Yam Richard, "An importance measure for multistate systems with external factors," Reliability Engineering and System Safety, vol. 167, pp. 49-57, 2017.

4. K. Hausken, and G. Levitin, "Review of systems defense and attack models", International Journal of Performability Engineering, vol. 8, no. 4, pp. 355-366, 2012

5. G. Levitin, H. Ben-Haim, "Importance of protections against intentional attacks", Reliability Engineering \& System Safety, vol. 93, pp. 639-646, 2008.

6. R. Peng, Q. Zhai, L. Xing, and J. Yang, "Reliability of 1-out-of-(n+1) Warm Standby Systems Subject to Fault Level Coverage", International Journal of Performability Engineering, vol. 9, no. 1, pp.117-120, 2013.

7. S. Si, H. Dui, Z. Cai, and S. Sun. "A novel importance measure for external factors based on system performance," International Journal of Performability Engineering, vol. 8, no. 4, pp. 447-450, 2012.

8. G. N. Taranto, T. M. L. Assis, D. M. Falcao, R. C. de Carvalho, "Highlighting the importance of chronology on voltage protection and control in active distribution networks", IEEE Transactions on Power Delivery, vol. 32, pp. 361-369, 2017.

9. L. Xing, G. Levitin, "Balancing theft and corruption threats by data partition in cloud system with independent server protection," Reliability Engineering \& System Safety, vol. 167, pp. 248-254, 2017.

10. Q. Zhai, Z. S. Ye, R. Peng, and W. Wang, "Defense and attack of performance-sharing common bus systems", European Journal of Operational Research, vol. 256, pp. 962-975, 2016.

Dui Hongyan is currently an Associate Professor in the School of Management Engineering, Zhengzhou University, Henan, China. He received his PhD (2013) from the School of Mechanical Engineering, Northwestern Polytechnical University, China. His research interests include importance analysis and system reliability. 